\title{
Les stratégies du discours publicitaire télévisuel algérien et français Étude des embrayeurs énonciatifs
}

\section{Résumé:}

Le discours publicitaire contemporain jouit d'une forme discursive particulière compte tenu de son principal objectif qui vise à capter l'attention d'un large public de plus en plus exigent. Cette contrainte permet alors de justifier le recours à plusieurs stratégies dans son organisation énonciatif, argumentatif, voire iconique. À cet effet, le but de cette étude est de repérer, d'interpréter et de comparer quelques stratégies énonciatives liées aux embrayeurs personnels et temporels dans la publicité télévisuelle algérienne et française.

Mots clés : discours publicitaire télévisuel, stratégies énonciatives, embrayeurs personnels et temporels.

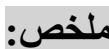

إن الخطاب الإعلاني المعاصر يملك شكل خطابي

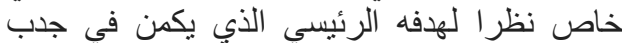
جمهور متطلب على نحو متز ايد. هذا القيد يبرد

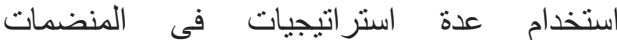
اللفظية،الجدلية، و حتى الليقونية. تحقيقا لهذه الغاية،

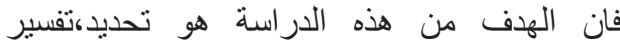
ومقارنة بعض الاستراتيجيات اللفظية المتعلقة

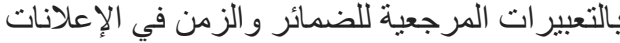
التلفزيونية الجز ائرية و الفرنسية.

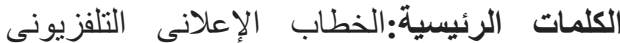

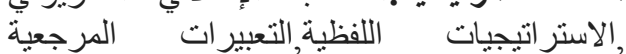
للضمائر و الزمن.

\section{HananeBENDIB}

Département des lettres et langue française Université des frères Mentouri

Constantine 1

\begin{abstract}
Introduction:
En publicité, les annonceurs font appel, lors de la rédaction de leurs discours publicitaires, à un langage spécifique qui nécessite l'usage de plusieurs stratégies énonciatives. Celles-ci se nouent au monde de la linguistique, de la rhétorique et de la pragmatique afin de créer des messages de plus en plus séduisants et attractifs. En effet, en misant sur la complicité et l'empathie, l'énonciation publicitaire englobe des stratégies discursives capables d'impliquer le public en l'incitant directement ou
\end{abstract}


Indirectement à l'adhésion de ce qui est énoncé. Notre perspective dans cette étude est de repérer, à partir d'un corpus médiatique composé de publicités diffusées dans des chaînes algériennes et d'autres françaises ; ces différentes stratégies énonciatives sont liées particulièrement aux embrayeurs. Ces derniers seront interprétés en fonction de leur contexte d'apparition (algérien et français). Ainsi, nous tenons à vérifier si le recours à ces stratégies de déictiques est lié ou non au contexte d'énonciation, à savoir le contexte algérien et français. En d'autres termes, nous tenons à répondre à la question principale suivante: le recours aux différentes stratégies des embrayeurs énonciatifs aurait-il une relation directe ou indirecte avec le contexte d'énonciation? En d'autres termes, les stratégies liées aux embrayeurs énonciatifs utilisées dans le discours publicitaire télévisuel algérien sont-elles identiques ou différentes de celles du discours publicitaire de l'hexagone ? L'hypothèse serait que les deux annonceurs (algériens ou français) recourent à des stratégies énonciatives identiques sur le plan sémantique, distinctives sur le plan syntaxique en fonction de leur visée énonciative.

Démarches méthodologiques, description du corpus et repères théoriques. Le recueil des données de l'ensemble du corpus s'est effectué selon deux méthodes : certaines publicités ont été enregistrées à partir des différentes chaînes algériennes et françaises publiques et privées; d'autres ont été téléchargées à partir des sites officiels des vidéos (You Tube et Dailymotion). Il s'agit de vingt (23) publicités différentes relatives aux produits de consommation quotidienne : des opérateurs de téléphonie mobile, des voitures, des produits alimentaires, des sponsors de sport, des spots de sensibilisation, etc. La sélection du corpus relève d'un hasard sain pour une analyse comparative fructueuse.

En ce qui concerne les repères théoriques, nous nous sommes orientée principalement vers les différentes méthodes que Maingueneau et Charaudeau proposent quant à l'interprétation des indices personnels et temporels. Ainsi, notre méthodologie à la fois interprétative et comparative vise principalement à travers une analyse du discours, à repérer, interpréter et comparer la fonction des embrayeurs énonciatifs présents dans le discours publicitaire télévisuel algérien et français.

Les embrayeurs comme stratégies énonciatives : interprétation des indices personnels et temporels.

Nécessaire de le rappeler, le concept d' "embrayeur » dérivé d'embrayage appelé également "déictique», "expressions sui-référentielles», «tokenreflexives 》 ou encore «symboles indexicaux », est une traduction française de $\mathrm{N}$. Ruwet du mot anglais «shifter ». Ce dernier, figurant chez Jespersen, est paru dans le vocabulaire de Jakobson, en 1963, qui l'utilise dans les quatre types de relations entre code et message, à savoir le message qui renvoie au code. 
Jakobson explique à ces propos que "la signification générale d'un embrayeur ne peut être définie en dehors d'une référence au message » [2]. Autrement dit, le contexte linguistique constitue, selon le linguiste, le repère par excellence de toute interprétation d'un embrayeur. C'est le cas des référents de tous les pronoms personnels ; par exemple, le «vous» dans un énoncé désigne un (des) destinataire(s) du message. Cependant, la pragmatique n'a pas vraiment partagé la vision de Jakobson quant à son expression de « référence ». Pour cette discipline, l'expression est usée dans une autre dimension, celle de la référence temporelle dans la pragmatique cognitive.

Notre étude sera donc consacrée à repérer les différentes catégories des embrayeurs figurant dans notre corpus et d'essayer de les analyser en prenant compte de la scène d'énonciation instituée par le discours tel que Maingueneau le préconise.

D'abord, il faut souligner que les embrayeurs présents dans le discours publicitaire et que nous avons repéré dans le corpus sont en particulier des pronoms personnels de la première et de la deuxième personne du singulier et du pluriel, à savoir le «je », «nous », 《tu » et « vous». D'autres pronoms y figurent également : il s'agit de la troisième personne (il, ils, elle, elles); et même des pronoms impersonnels (il, on) à des degrés d'aspérité. Cependant, les pronoms personnels sujets ne sont pas les seuls déictiques. Le discours publicitaire en regroupe d'autres catégories : des possessifs, des démonstratifs, des adverbes, des locutions adverbiales locatives et temporelles, des catégories du présent, du passé et du futur.

\section{Catégorie des indices personnels.}

\section{Le « Nous » et le « vous ».}

Ce qui a retenu notre attention, c'est le fait que ces deux pronoms (nous, vous) sont présents avec une fréquence assez remarquable. En effet, sur le nombre de vingt trois publicités, le «vous » et le «nous » sont exploités dans quinze (15) sur l'ensemble du corpus, ce qui représente une fréquence de $65 \%$. Cet usage n'est pas vraiment nouveau dans les discours ayant un large public pour destinataire, le cas de la publicité.

Le « Nous », en réalité, ne se réfère pas à un groupe mais plutôt à une instance collective. Benveniste explique, dans ce sens, que «d'une manière générale, la personne verbale au pluriel exprime une personne amplifiée et diffuse » [3].

Dans la publicité, nous pourrions avancer l'hypothèse que derrière ce "Nous» se cache une marque et derrière cette marque se cache un groupe d'individus qui sont à la source de cette marque, nous parlons ipso facto de l'annonceur (l'entreprise de la marque). Le «vous» serait lié de sa part au public, à qui le message est destiné. Cependant, dans certains spots publicitaires, les deux pronoms, se trouvant dans l'énonciation même, 
n'indiquent en aucun cas qu'il s'agit d'un fabricant qui s'adresse à un public. Ainsi nous retrouvons-nous dans une confusion de personnes.

Considérons des exemples de notre corpus médiatique et essayons de présenter une interprétation des embrayeurs de personnes.

L'embrayeur « Nous ».

(1) "Aujourd'hui dans le tramway d'Alger, nous sommes réunis" (pub1 /contexte algérien)

(2) «Réseau ferré de France: nous avons de grands projets pour vous »(pub2/contexte français)

(3) "Quoi qu'il arrive, nous resterons toujours debout» (pub3/contexte algérien)

(4) «Les athlètes sont la lumière des jeux. Nous sommes fières de les éclairer. " (pub4/contexte français)

(5) "Il nous faudra agir pour une route plus sûre.»(pub5/contexte français)

Dans le premier texte (pub1/contexte algérien), le message est émis par un personnage connu de la société algérienne, un spécialiste du domaine de la route et de la sécurité routière. Le spot publicitaire a pour thème la mise en service d'un nouveau moyen de transports publics (tramway) dans la ville d'Alger. Le scénario réunit plusieurs personnages représentant les citoyens algérois qui décident d'emprunter ce moyen de transport qui semblerait faciliter le quotidien de plusieurs d'entre eux.

À cet effet, l'usage du « Nous » à la fin de la publicité ne serait, selon Maingeuneau, qu'une stratégie pour valoriser «la face positive » du public qui serait davantage concerné par le message. Il serait inadéquat de dire « Aujourd'hui dans le tramway d'Alger, vous êtes réunis », car dans ce sens, le public aurait une mauvaise interprétation quant à l'utilisation de ce moyen de transport. Ainsi, la «face positive » du destinataire serait dévalorisée amenant le public à penser qu'il s'agit d'un moyen destiné à une couche sociale déterminée (des personnes qui ne possèdent pas de voitures, par exemple). Cette «face négative » pourrait éventuellement engendrer des « représentations discriminatoires » de la part du public l'incitant à réfuter l'idée d'abandonner la voiture et d'emprunter ce nouveau moyen de transport. Ainsi, le « Nous », associé à un présent dans cet extrait, implique à la fois l'annonceur (entreprise Itousa) et le public (téléspectateur algérois).

Dans le second exemple (pub2/contexte français), le « Nous» ne se présente pas de la même manière que dans le premier extrait du fait qu'il ya un autre embrayeur, à savoir le "Vous ». Cela écarte d'ores et déjà l'idée du «Nous» qui rassemble l'annonceur et le public. Dans cet énoncé, les deux sujets sont séparés : un « Nous » pour l'annonceur (Réseau ferré de France) et «Vous » pour le récepteur (les téléspectateurs français). Cette distinction, dans l'usage, sépare ainsi les sujets de la communication mettant de côté chaque 
élément à part. C'est de cette manière que l'annonceur arrive à transmettre ses promesses à son public : "nous, l'entreprise du réseau ferré de France, avons de grands projets pour vous, peuple de France $»$.

L'embrayeur "Nous» dans le troisième énoncé (pub3/contexte algérien) fonctionne de la même façon que le premier extrait. C'est-à-dire ; il implique l'annonceur et le public. Le message est un extrait d'une chanson diffusée pour un sponsor d'un événement sportif qui implique l'équipe nationale du football algérien et leurs supporters. Le scénario réunit les anciens joueurs de l'équipe algérienne avec ceux de la nouvelle génération. Ce "Nous» pourrait donc projeter l'image du sponsor officiel (opérateur de téléphonie mobile Nedjma), les sportifs algériens et leur public.

Pour le quatrième extrait (pub4/contexte français), le «Nous» se positionne d'une manière claire du côté de l'annonceur. Il renvoie clairement à l'instance énonciatrice, à savoir l'Entreprise d'Électricité de France (EDF) qui exprime son soutien quant à la participation des athlètes français aux Jeux Olympiques organisés à Londres. Toutefois le public, dans une énonciation à la modalité élocutive, voire à caractère "performatif» [1], n'occupe que le statut d'un simple «témoin » de l'acte de proclamation du publicitaire.

Quant au dernier exemple (pub5/contexte français), le «Nous» implique clairement les deux parties de la communication, car il s'agit d'un message de sensibilisation pour les accidents routiers. Comme l'émetteur, le destinataire est aussi responsable de la sécurité routière où chacun est appelé à accomplir son devoir de citoyen.

Dans l'ensemble des extraits que nous avons relevés, le «Nous» publicitaire est lié à un présent non déictique qui masque la fonction réelle de la publicité qui peut être : une proposition, un conseil, voire une incitation pour l'achat d'un produit. En d'autres termes, le locuteur expose ses offres dans son propre monde virtuel, celui de la publicité. Il en est le cas de plusieurs énoncés non embrayés où le présent des verbes cache parfois une prescription (la notice), un devoir faire (les récits), explique Maingueneau.

L'embrayeur « Vous » :

(1) «...Allo OTA vous offre cinquante pour cent de crédit supplémentaire... » (pub 6/contexte algérien)

(2) «SFR carrément vous" (pub7/contexte français)

(3) «Bouygues télécom en faire plus pour vous » (pub 8/contexte français)

(4) «Moi j'ai choisi le shampoing Vénus deux en unet vous?" (pub 9/contexte algérien)

(5) "Vous aimez vos cheveux?» (pub10/contexte français)

(6) "Qu'attendez-vous de la nouvelle génération?" (pub11/contexte français) 
(7) «Personnage $1 H$ :bonjour, vous n'auriez pas vu notre ballon?» (pub 12/contexte français)

(8) «Personnage $2(H)$ :ben dans le pack de MAAF c'est écrit// vous pouvez nous évaluer : content pas content, c'est vous qui l' dîtes» (pub 13/contexte français)

(9) «vous pouvez gagner » (pub14/contexte algérien)

(10) «vous à la maison [...]» (pub 15/contexte français)

Dans les dix extraits, le destinataire est présent à travers la forme d'un vous (tu) et désigne respectivement non pas un seul individu mais un groupe selon le type du message : des utilisateurs de la téléphonie mobile, des femmes soucieuses de la santé et de l'entretien de leurs cheveux, des fans de la nouvelle technologie, des personnes en quête de voitures de qualité, un public qui cherche une meilleure assurance et des téléspectateurs passionnés par les jeux de culture. Chacun d'entre eux est désigné par le pronom personnel « Vous ».

Dans le premier, le deuxième, le troisième et le huitième exemple le locuteur, effacé et caché derrière une entreprise (des opérateurs de téléphonie mobile: Allo, SFR, Bouygues Télécom), présente son offre au destinataire désigné par le pronom «vous». Ce dernier s'identifie au «vous», une stratégie de la part de l'annonceur qui tente d'établir un contact de confiance afin que chaque téléspectateur se voit dans cette offre et s'identifie à cette marque.

Dans d'autres cas, Charaudeau nous parle dans les textes publicitaires de la présence d'un dialogue simulé entre un locuteur et un interlocuteur fictif sous une sorte de "contrat de confiance »; ce dialogue se fait à travers une mise en scène dans laquelle le locuteur est un personnage qui apparaît via un pronom personnel « je, moi » et s'adresse au public (éventuel consommateur), comme dans le quatrième exemple «Moi, $\boldsymbol{j}$ 'ai choisi le shampoing Vénus deux en unet vous? », voire dans l'exemple six : «qu'attendez-vous de la nouvelle génération?».

Cette situation de dialogue simulé ne se présente pas toujours de la manière que nous venons de voir dans l'exemple quatre où les personnages du dialogue sont en l'occurrence l'annonceur et le public. Il existe, en réalité, ce que Charaudeau appelle "dialogue intimité » dans lequel il expose des personnages qui jouent des rôles spécifiques selon un scénario. Le public est sensé se sentir concerné par le message et se met à la place du bénéficiaire de l'action du produit après que son désir serait exhaussé.

Les extraits sept et huit font partie de ce modèle de dialogue intimité. Dans le huitième exemple, une discussion, ayant pour thème les qualités d'un service d'assurance de l'entreprise la MAAF, expose des soucis susceptibles d'être évoqués par les utilisateurs de cette compagnie, pour ceux qui veulent l'adopter et s'interrogent sur ses différentes offres et même pour ceux qui ne la 


\section{Les stratégies du discours publicitaire télévisuel algérien et français}

Étude des embrayeurs énonciatifs

connaissent pas encore. L'un des personnages de ce dialogue, à qui le public est censé s'identifier, montre clairement son mécontentement à l'égard de cette compagnie et cherche la moindre faille dans son système. L'autre personnage, ayant pour rôle le Directeur de l'entreprise, tente de rassurer ce client "affolé ». Le Directeur réussit sa mission en s'appuyant sur des arguments convaincants grâce à la nouvelle procédure de la compagnie : un baromètre satisfaction au moyen duquel les abonnés peuvent évaluer constamment la compagnie dans le but d'une éventuelle amélioration.

Le « Je ».

Prenons les extraits publicitaires où le pronom personnel «Je» constitue le sujet principal de l'énonciation :

(1) Hé! Inspecteur, apparemment cette femme est dépassée par " ramadhan " je vais les appeler (pub 6/contexte algérien)

(2) « moi, c'est ce que je ressens quand j'entends encore dire qu'on a pas un bon réseau chez Bouygues télécom 》. (pub 8/contexte français)

(3) " j'étais au pied du sapin(...) je m'étais mis sur mon trente et un, enfin je veux dire sur mon vingt cinq(...) là ils ouvrent leurs cadeaux et hop un téléphone et hop encore un téléphone et encore un autre, ça n'arrêtait plus. Alors sur le coup j'ai cru que c'était un coup de show, que j'avais diversion mais en fait pas du tout, c'était une sorte de super cadeau commun. Oh! à les voir tous réunis, ça m'a fait fondre " (pub16/contexte français)

(4) Café Mani, $\boldsymbol{j}$ 'aime son goût (pub 17/contexte algérien)

(5) "Moi, au mois de ramadhan ma demeure s'épanouit quand je suis entourée de mes enfants, je leur prépare tout ce qu'ils aiment; et avec tous ces repas, je me sentais toujours ballonnée, je croyais que c'était normal à mon âge, mais lorsque j'ai essayé ACTIVIA, le ballonnement m'est devenu anormal » (pub 18/contexte algérien)

(6) "Ah! franchement je confirme ; celui aux mangues là. J'aime beaucoup " (pub 19/contexte français)

(7) «je peux contrôler sa température comme je veux, et tout produit que tu mets dedans reste frais » (pub 20/contexte algérien)

(8) «oui, je l'aurais un jour, je l'aurais » (pub 13/contexte français)

(9) «Personnage 1 (H): c'est bon vous êtes prêts, je vous accompagne? (pub 1/contexte algérien) »

(10) "Je crois toujours en toi, en avant je te suis» (pub 3/contexte algérien)

Si nous observons de près les extraits ci-dessus, nous pouvons voir clairement que le pronom personnel « Je » n'est pas utilisé de la même manière dans chaque exemple. Parfois, le pronom est intégré dans le dialogue interne du 
discours publicitaire entre les personnages de la publicité (pub 6, 20,13 et 1) ; dans d'autres le «Je» a pour rôle d' «identification »[3]. Autrement dit, le téléspectateur s'identifie à l'instance énonciatrice mise en scène. Dans la première partie de la publicité 18: "Moi, au mois de ramadan ma demeure s'épanouit quand je suis entourée de mes enfants, je leur prépare tout ce qu'ils aiment; et avec tous ces repas je me sentais toujours ballonnée, je croyais que c'était normal à mon âge»; le "Je» témoigne d'une situation susceptible d'être vécue par chaque femme en ce mois de «Ramadhan » (un mois hégirien sacré de jeûne chez les musulmans ); un malaise auquel les téléspectatrices en particulier souhaiteraient trouver une solution. En effet, le personnage (une femme d'âge moyen) apporte cette solution dans la deuxième partie de l'énonciation: «mais lorsque j'ai essayé ACTIVIA, le ballonnement m'est devenu anormal ». Ce « je » singulier vise donc à inclure toute téléspectatrice chez qui l'on suscite cette identification indirecte puisque celle qui se trouve devant son téléviseur devrait d'abord sentir qu'elle est concernée par le message.

Un autre type de « je » qui n'implique pas le processus d'identification. Il s'agit plutôt d'un « Je » énonciatif de la part d'un locuteur énonciatif destiné à un «tu » récepteur (le public) dans une situation de dialogue simulé; exemple «moi, c'est ce que je ressens quand j'entends encore dire qu'on n'a pas un bon réseau chez Bouygues Télécom ». Le locuteur (ingénieur en réseau) exprime à travers ce propos son mécontentement envers ceux qui critiquent les services de la téléphonie mobile Bouygues Télécom. Il compare ses sentiments à un cri d'animal (grognement d'un ours) pour montrer sa « révolte» au public destinataire. Ce dernier pourrait éventuellement changer son comportement et ses représentations négatives- s'il en est le cas- en jugement positif.

Il existe également un autre genre de «Je» qui apparait dans la seizième publicité que nous pourrions désigner par un «Je narratif». Le locuteur (personnage fictif) raconte son expérience annuelle pour les fêtes de Noël pendant lesquelles il est témoin des offres tentantes de l'opérateur Orange. Ici, l'énonciateur accomplit une double fonction : il informe d'une part le téléspectateur de ces offres et, vise d'autre part à provoquer une identification chez le public par le propos : «Oh ! À les voir tous réunis, ça m'a fait fondre $»$. Le téléspectateur, n'ayant pas choisi l'offre, pourrait subir le même sort du personnage de la bougie (exprimé métaphoriquement par «ça m'a fait fondre »). Ainsi, il risquerait de manquer ces offres séduisantes.

Maingeuneau parle également d'un «Je» qui a une "place vide et qui peut être assumée par n'importe qui» [3]. Dans ce cas le téléspectateur concerné par le message est qualifié pour occuper cette place vide du « je » qui est aussi l'agent de l'action exprimé par le verbe de la phrase. C'est le cas de la pub 17 "Café Mani, j'aime son goût» et la pub 3 «Je crois toujours en toi, en avant je te suis ». Le public, n'ayant jamais ou ayant déjà goûté au café Many, 
pourrait occuper ce «je». De même chaque supporteur, concerné par cet événement,de l'Équipe nationale du football algérien.

Le « On » et le « II/Ils».

En plus des pronoms personnels «je, nous et vous » qui se réfèrent à l'énonciateur et au co-énonciateur, d'autres pronoms constituent également les sujets de l'énonciation publicitaire. Il s'agit des pronoms : «Il/ils et On » qui présentent un certain nombre de caractéristiques selon le contexte textuel d'énonciation. Dès lors, si nous voulions comprendre l'emploi de ces embrayeurs, il serait plus plausible d'observer quelques extraits afin de déterminer la fonction, plus ou moins, exacte de ces pronoms selon leur contexte textuel d'apparition. D'ailleurs, Maingueneau parle de rôle «polyvalent» du «On » qui lui permet d'être au centre de diverses stratégies énonciatives. Charaudeau, de son côté, attribue au " On» le rôle de «tiers » dans lequel les trois parties de l'énonciation (locuteur, personnage et destinataire) peuvent se projeter.

La présence du «On» n'est pas l'embrayage impersonnel unique en publicité. En effet, le «il/ils» constitue une autre figure de cette forme d'embrayage. Ces outils sont utilisés pour se référer à un sujet indéterminé appelé « collectif» [3].

Voici deux extraits qui comportent les embrayeurs « On » et «il/ils » :

(1) « (...) là ils ouvrent leurs cadeaux (...) on pourrait au moins rester en contact, pas vrai les gars?" (pub16/contexte français)

(2) « (...) on n'en sort pas de table près d'un mois /du coup après on se sent un peu ballonner. Alors on a essayé le programme Activia (...)» (pub 19/contexte français)

Dans la pub 16, le pronom indéfini collectif «Ils », en se référant au contexte textuel, renvoie aux personnages dont le personnage-locuteur (la bougie du père noël) nous en fait une description. Il s'agit en l'occurrence, des membres de la famille et amis chez qui « la bougie » est témoin de l'événement en question. Dans l'énoncé, le «ils » n'a aucun antécédent et pourtant cela ne nous a pas empêché de déduire les sujets référents grâce au contexte textuel d'énonciation. Ensuite, et à la fin du texte, le personnage-locuteur utilise cette fois-ci le «On» qui se réfère à lui et à ses amis (d'autres bougies) d'une part. D'autre part, l'effacement du « Nous » est dû sans doute à la polyvalence de ce pronom; ainsi créé-il implicitement une forme d'identification de la part du destinataire téléspectateur qui se projetterait à son tour dans cette situation.

Pour le second extrait, l'énonciationdu locuteur personnage oscille entre le «je» et le «on». Dans ce sens, l'usage du «on» n'est qu'une référence au «nous» dans un registre non formel. Le locuteur personnage, en fait, a eu recours au « On », car il s'agit d'un témoignage à l'oral dans un style « décontracté » offrant au destinataire un contexte textuel entièrement familier auquel il peut facilement s'identifier. 


\section{Des textes sans personnes.}

Nous venons d'examiner une série d'exemples de discours publicitaire qui comportent divers embrayeurs de personne. Dans d'autres, l'énonciation s'effectue par un procédé appelé " effacement des embrayeurs de personne " [3]. Il s'agit, entre autres, d'une stratégie énonciative qui vise à adhérer pleinement le public en l'invitant à approuver la véracité du propos. Prenons sous cette optique cet extrait de notre corpus :

«Révolution L'Oréal ELSEVE Arginine Resist, découverte majeure : l'arginine protéine indispensable à la croissance du cheveu pour une triple action renforçatrice: 1) nutrition du bulbe, 2) renfort dès la racine, 3) restructuration de la fibre. Les cheveux poussent plus résistants. Nouvel ELSEVE arginine Resist de l'OREAL Paris ".

Voix off $F$ : Quatre-vingt-quinze pour cent des femmes ont constaté son efficacité. (pub10/contexte français)

Cet extrait témoigne clairement de l'absence des deux parties de la communication (énonciateur et Co-énonciateur). Aucune trace d'embrayeur n'y figure. Le texte ne répond pas alors à la structure d'énonciation ordinaire que nous avons préalablement constatée, car il fait partie du plan "non-embrayé » qui « est liée à un parasitage des caractéristiques du discours scientifique »[3]. Cette forme d'énonciation semble exposer le nouveau shampoing de la marque ELSEVE de L'OREAL comme une « vérité scientifique », indépendamment du destinateur et du destinataire. Ce caractère de scientificité apparaît nettement lorsque l'on parle d'abord de la découverte de «l'Arginine », une protéine nécessaire pour le bon entretien des cheveux. Ensuite de ses trois actions bénéfiques, le résultat promis «Les cheveux poussent plus résistants » et enfin le pourcentage des femmes qui ont constaté l'efficacité du produit présenté à la fin du texte.

Cependant, il est nécessaire de souligner que la téléspectatrice qui n'est citée que par une partie d'elle "ses cheveux », est impliquée indirectement dans cette atmosphère qui répond à ses besoins esthétiques d'avoir des cheveux qui poussent en bonne santé ; et qui est d'ailleurs le souci des autres femmes qui s'identifient automatiquement à l'énoncé de : «quatre-vingt-quinze pour cent des femmes ont constaté son efficacité ».

Les indices temporels : place et rôles des modes et des temps.

Comme tous discours à pouvoir incitatif, l'impératif constitue le mode $\mathrm{du}$ discours publicitaire au premier niveau, suivi des autres modes qui varient entre indicatif et infinitif. Ce constat de modes est principalement présent dans le contexte français par rapport au contexte algérien.

En effet, concernant le discours publicitaire algérien, repérer et classer les modes, semble une tâche délicate, car les langues présentes dans ce contexte se présentent dans une situation plurilingue. D’une part l'arabe " algérien », en plus de l'arabe « scolaire » sont les langues les plus utilisées; d'autre part le 
français et d'autres langues (l'anglais en particulier) sont moins présentes dans ces textes, créant ainsi un discours à caractère plurilingue. En effet, ce multilinguisme dans le contexte algérien pourrait être considéré comme une stratégie discursive qui contribue davantage à créer un discours publicitaire reflétant les pratiques langagières d'un public plurilingue.

De ce fait, nous avons opté pour la traduction littérale des textes à expression arabe pour pouvoir, plus ou moins, constituer une liste qui comporte uniquement les verbes à expression arabe qui indiquent des temps différents, pouvant nous mener à des résultats plus ou moins représentatifs :

\begin{tabular}{|c|c|c|c|}
\hline Publicité & $\begin{array}{ll}\text { Verbe en } & \text { arabe } \\
\text { (algérien/scolaire) } & \end{array}$ & $\begin{array}{l}\text { Traduction } \\
\text { littérale en } \\
\text { français }\end{array}$ & $\begin{array}{l}\text { Temps et } \\
\text { modes estimés }\end{array}$ \\
\hline 21 & 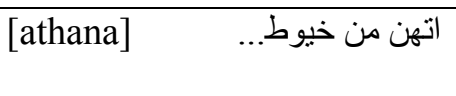 & $\begin{array}{l}\text { Débarrasse- toi } \\
\text { des câbles... }\end{array}$ & Impératif \\
\hline 6 & غ8labha ramdan] غلبها رمضان & $\begin{array}{ll}\text { Elle } & \text { est } \\
\text { surmontée } & \text { par } \\
\text { Ramadhan } & \\
\end{array}$ & Passé \\
\hline 6 & $\begin{array}{l}\text { Allo OTA [tuhdikom } \\
\text { xamsin bil mipa] } \\
\\
\\
\text { تهديكم خمسين بالمائة }\end{array}$ & $\begin{array}{l}\text { Allo OTA vous } \\
\text { offre cinquante } \\
\text { pour cent }\end{array}$ & Présent \\
\hline 9 & [ba $\int$ nsagmu] & $\begin{array}{l}\text { Pour que nous } \\
\text { réparerions }\end{array}$ & $\begin{array}{l}\text { Futur } \\
\text { (subjonctif) }\end{array}$ \\
\hline
\end{tabular}

D'abord, il faut signaler que les verbes en grammaire arabe sont exprimés en trois principaux groupes de temps et de modes : 1) le passé, 2) le présent/le futur et 4) l'impératif.

Ainsi retrouvons-nous cette classification dans les textes analysés même si la plupart d'entre eux sont exprimés en arabe "algérien ». D'ailleurs, lors de la classification que nous avons établie dans le tableau ci-dessus, nous avons pu recenser quatre formes de verbes (impératif, passé, présent, futur) auxquels nous avons tenté, en se basant sur la grammaire arabe, de présenter une traduction en langue française en respectant au mieux les modes et les temps.

Après avoir repéré les principaux temps et modes dans l'énonciation publicitaire à expression arabe, essayons à présent de les interpréter selon leurs deux contextes d'apparition (algérien et français) pour tenter en outre de repérer d'autres stratégies énonciatives.

\section{Le mode impératif.}

Que ce soit dans le contexte français ou algérien, l'impératif occupe une place prépondérante au sein du discours publicitaire. Ce mode, en réalité, 
n'a pas la valeur d'une injonction directe ; au contraire il s'agit d'une stratégie discursive de la part du publicitaire pour se rapprocher davantage du consommateur du fait que ce mode est souvent utilisé entre amis, à travers plusieurs modalités: une proposition, un conseil, une suggestion, une interdiction, etc. En effet, c'est de la part d'un ami que l'on reçoit souvent des propositions, des conseils, des suggestions ou encore des interdictions exprimées par des verbes au mode impératif.

\section{Le mode indicatif.}

Il vrai que le mode indicatif n'existe pas dans la conjugaison de la langue arabe à la manière de la langue française ; mais son usage est présenté par des temps tels que le présent et le passé.

\section{Le présent.}

Dans certains énoncés publicitaires, les embrayeurs de personne sont associés à un présent non déictique dans un extrait non embrayé, valides pendant un temps et un espace limités (plus ou moins durant la disponibilité du produit sur le marché). Ce choix de temps sous-entendrait généralement :

$>$ Une description: "Le réfrigérateur \{side by side\} ENIEM de deux fonctions comportant une fontaine d'eau fraîche, un distributeur automatique des glaçons (...) On peut retirer les boissons sans ouvrir le réfrigérateur et regarde ce tiroir magique, je peux contrôler sa température comme je veux et tout les produit que tu mets dedans restent frais » (pub 20)

> Une explication: "Grâce au bifidus Acti-régularise, ACTIVIA aide à combattre le ballonnement $\gg$ (pub 18)

$>$ Une information: "le personnel des agences commerciales demeure à votre disposition pour toutes les informations complémentaires » (pub21/contexte algérien)

$>$ Une narration: «(...) moi, les copains je les vois qu'une fois par an et encore pas longtemps (...) 》(pub 16)

$>$ Une suggestion: «Mobilis, et tout le monde parle»(pub22/contexte algérien)

\section{Le passé (le passé composé et l'imparfait)}

Le temps passé dans le genre publicitaire pourrait connoter principalement « un savoir-faire » de l'annonceur, grâce auquel le public aurait plus de confiance. Corollaire, le public se sentirait mieux rassurer en pensant que ce produit a fait l'objet de plusieurs essais avant que l'on lui propose. Une téléspectatrice songerait, par exemple, à essayer le nouveau shampoing de L'OREAL après avoir appris que « quatre-vingt-quinze pour cent des femmes ont constaté son efficacité »".

Quant à l'imparfait dans le discours publicitaire, il se réfère plutôt au caractère narratif que peut comporter ce genre de discours. Exemple : «j'étais au pied du sapin, pas trop près pour éviter les dégâts. Je m'étais mis sur mon 
trente et un, enfin je veux dire sur mon vingt cinq » (pub 16). Le personnage de la pub relate les circonstances de l'événement (une soirée de Noël) pour qu'un manque se déclenche automatiquement chez le téléspectateur qui ressent le besoin de se procurer l'objet du spot publicitaire.

\section{Le futur.}

Avoir recours au futur dans l'énonciation publicitaire véhicule ordinairement un engagement de la part de l'annonceur. D'ailleurs, ce constat est largement appuyé par la modalité élocutive de promesse ou encore dans les actes «promissifs»[4]initiés par Searle dans lesquels le locuteur est amené à réaliser une certaine conduite au futur.

En parcourant les différents énoncés du discours publicitaire télévisuel du contexte français de notre corpus dans le but de relever des extraits comportant des verbes conjugués au futur, nous sommes parvenue au constat que le discours publicitaire n'utilise pas ce temps de la manière que la conjugaison le dicte. L'annonceur préfère plutôt une forme plus anodine pour exprimer ses promesses et ses engagements, c'est le cas de cet extrait de la publicité 11 où l'annonceur use de la formule : aller au présent + verbe à l'infinitif: "LED tv la génération qui va tout changer ". D'ailleurs, ce choix pourrait bien être justifié par la valeur même de cette formule qui exprime temporellement le futur proche; une manière de rassurer les téléspectateurs quant à l'avènement proche de l'offre de l'annonceur.

Dans d'autres cas, le futur n'exprime pas toujours les promesses, ni les engagements de l'annonceur; il s'agit simplement de transmettre des faits futurs. Ce temps futur est notamment utilisé dans les annonces à visée informatrice et sensibilisatrice comme pour cet extrait de la pub 5: «Tant qu'il y aura trop d'alcool dans le sang d'un conducteur(...)». Dans cet exemple, l'annonceur ne s'engage pas et ne promet rien, il ne fait qu'avancer des constats évidents dans le but d'affirmer ses propos pour une meilleure sensibilisation.

En ce qui concerne les publicités du contexte algérien, la présence du futur n'est pas très distinctive du fait que la grammaire arabe ne compte que deux formes de temps verbales: le passé et une forme commune au temps présent et au temps futur. Toutefois, cela n'empêche pas l'annonceur d'exprimer ses promesses et ses engagements comme pour ce slogan de la pub 23 de la lessive OMO : 《يوسن حوايجو ويزيديتعلمهو المفيد "). Les deux verbes en gras et en italique qui signifient respectivement «salir » et «apprendre » sont conjugués au futur selon la grammaire arabe. 


\section{Le mode infinitif.}

En observant les textes de notre corpus, il s'avère que l'infinitif employé seul n'est pas un mode très fréquent dans le discours publicitaire par rapport à l'impératif qui constitue le mode de ce discours par excellence. Le seul extrait où nous avons repéré l'infinitif est dans le slogan de la publicité 8 de l'opérateur Bouygues Telecom: «Bouygues Télécom, en faire plus pour vous! ».

Cette faible présence du mode infinitif dans le discours publicitaire pourrait bien être justifiée par la valeur même de ce mode. En effet, employé seul, l'infinitif comporte la valeur d'un ordre ou d'un conseil. Il représente donc un mode redondant de l'impératif. Or, les annonceurs optent plutôt pour l'impératif connu pour ses valeurs de conseil et d'incitation à la place de l'infinitif qui est moins connu pour cet usage.

Ainsi opèrent les modes et les temps dans le discours publicitaire. On y trouve en premier lieu et à une grande échelle l'usage de l'impératif comme une stratégie discursive de type amical dans le but de se rapprocher davantage du public.

Ensuite, vient l'indicatif dans lequel nous retrouvons le présent qui comporte des valeurs d'ordre descriptif, explicatif, informatif, narratif et suggestif; le passé composé quant à lui connote plutôt le «savoir- faire» de l'annonceur afin de créer une certaine confiance chez le public; l'imparfait renvoie à la narration et le futur se charge d'assurer l'engagement et la promesse du publicitaire.

Enfin et en dernier lieu nous retrouvons une présence infime du mode infinitif employé seul qui comporte les mêmes valeurs que l'impératif.

\section{Conclusions.}

L'interprétation des embrayeurs personnels dans l'énonciation publicitaire nous a révélé que les pronoms « Nous » et «Vous» sont pleinement exploités dans la pub. Ils ne se réfèrent pas à un groupe mais à une instance collective.

Même si à l'évidence ces pronoms renvoient respectivement à l'annonceur et au public, on les retrouve à l'intérieur de l'énonciation sans aucun indice pouvant déduire cette évidence. Cette technique renvoie, à l'évidence, aux caractéristiques énonciatives du discours publicitaire, parmi lesquelles on retrouve la valorisation de la face positive du public. Ce dernier pleinement impliqué dans le discours soit à travers le « Nous », soit à travers le «Vous ». Dans le cas du « Vous » les deux instances, à savoir l'annonceur et le public, sont désignées séparément dans l'énonciation.

Dans d'autres cas, le public est écarté et n'occupe la place que d'un simple témoin dans l'énonciation. Ce statut de témoin n'évoque pas toujours son absence puisque dans d'autres circonstances le téléspectateur, même si le 
texte ne comporte aucun indice personnel à son égard, est censé s'identifier aux personnages représentant sa situation.

Par ailleurs et en ce qui concerne le pronom «Je", nous sommes parvenue à repérer cinq types de ce pronoms dans l'ensemble du corpus : 1) un $J e$ "intégré » dans le scénario du discours qui opère entre les personnages de la publicité ; 2) un $J e$ "d'indentification » qui implique le téléspectateur en fonction de ses besoins ; 3 ) un Je "énonciateur » qui renvoie simplement au publicitaire qui simule à son tour un dialogue en s'adressant à un $T u$ récepteur (le public) ; 4) un Je narratif employé par un personnage qui relate un fait ; et enfin 5) un $J e$ "vide» que peut occuper n'importe quel téléspectateur susceptible d'être concerné par le fait présenté.

Nous avons cependant repéré des textes publicitaires dans lesquels des pronoms indéfinis, impersonnels et personnels, comme «On » et « $\mathrm{Il} / \mathrm{ils}$ », côtoient les pronoms personnels «Je, Nous et Vous». Ceux-ci occupent des fonctions selon le contexte textuel de leur apparition. Le «On» remplit la fonction d'un pronom à la fois polyvalent et tiers. Ce double rôle lui permet d'être au centre de diverses stratégies discursives telles que la capacité d'impliquer en même temps l'annonceur, les personnages et le public, ou encore la possibilité de remplacer simplement le «Nous» dans un registre non formel. Quant aux «Il/ils», ils se réfèrent respectivement à un sujet indéterminé singulier ou collectif repérable à l'aide du contexte textuel.

Il existe en outre, des textes dépourvus de pronoms personnels qui possèdent parfois le caractère de scientificité, une stratégie énonciative qui vise à impliquer le public et à le faire adhérer à la véracité du discours à l'aide des outils énonciatifs outre que les embrayeurs personnels.

Par ailleurs, l'interprétation des indices temporels dans le discours publicitaire révèle que l'usage amplifié de l'impératif sous-entendrait en réalité la présence d'une stratégie discursive «amicale» employée dans le but de se rapprocher du public.

En outre, le mode indicatif est moins utilisé. Il englobe : 1) le présent qui comporte des valeurs d'ordre descriptif, explicatif, informatif, narratif et suggestif ; 2) le passé composé qui renvoie plutôt au "savoir-faire" de l'annonceur dans le but d'assurer sa place auprès du public en tant que spécialiste du domaine ; 3) l'imparfait qui renvoie à la narration et 4) le futur qui véhicule l'engagement et la promesse du publicitaire.

En dernier lieu et avec une utilisation minime, le mode infinitif employé seul comporte dans le discours publicitaire analysé les mêmes valeurs que l'impératif dans la mesure où il pourrait s'agir encore d'une stratégie énonciative qui repose essentiellement sur la diversification des modes et des temps.

Aussi retrouvons-nous d'autres stratégies énonciatives d'ordre temporel à travers lesquelles l'annonceur joue sur leur valeur pour se 
rapprocher de son public et cela à travers l'impératif, l'indicatif ou encore l'infinitif.

Par ailleurs et en ce qui concerne le contexte d'apparition, nous retrouvons plusieurs stratégies énonciatives communes aux deux contextes analysés. En effet, que ce soit dans le contexte français ou algérien, la stratégie de la mise en valeur de la face positive du public est présenteà travers l'emploi du pronom « Nous » en français ou «نا 》 en arabe.

En sus, l'étude montre que la stratégie qui repose sur la polyvalence du pronom « On» en français est semblable à celle du pronom « حنا 》 en arabe.

Se rajoute à cette troisième stratégie, le processus d'identification présent dans les textes sans pronoms. Celui-ci est employé par les deux contextes analysés en fonction de la visée principale du message publicitaire.

En plus, la stratégie de la diversité des valeurs du pronom «Je » est aussi utilisée par le contexte français que par le contexte algérien.

En dernier lieu, nous pourrions admettre que la quatrième stratégie de la diversité des temps et des modes est à son tour une caractéristique de chaque énonciation publicitaire des deux contextes approchés. Il est à retenir à cet effet que les deux discours publicitaires qu'ils soient algériens ou français recourent en premier degré à l'impératif qui occupe une place prépondérante suivie des autres modes et temps qui véhiculent des valeurs en fonction de l'objectif principal du message publicitaire.

\section{Bibliographie.}

[1] AUSTIN, J. L., 1970, trad. française par G. LANE, Quant dire c'est faire, éd. Seuil, p.26

[2] CHARAUDEAU, P, MAINGUENEAU, D, 2002, Dictionnaire d'analyse du discours, éd. Seuil, Paris, p.212,

[3] MAINGUENEAU, D., 2014, Analyser les textes de communication, éd. Armand Colin, Paris, p.32,33, 131, 134, 135, 139.

[4] SEARLE, J.R., 1982, traduction par J. Proust, Sens et expression, études de théorie des actes de langage, éd. Minuit, Paris, p154.

\section{Corpus médiatique.}

\section{Conventions de la transcription phonétique :}

Le tableau présente l'ensemble des caractères de la transcription phonétique utilisés lors de la transcription de notre corpus médiatique. Par la suite nous 
Les stratégies du discours publicitaire télévisuel algérien et français

Étude des embrayeurs énonciatifs

avons présenté la signification de chaque procédé dont nous nous sommes servie pendant la transcription de chaque publicité.

\begin{tabular}{|c|c|c|c|}
\hline Caractère arabe & Caractère latin & Caractère arabe & Caractère latin \\
\hline$\varepsilon / 1$ & \begin{tabular}{|l|}
5 \\
\end{tabular} & س & $\mathbf{S}$ \\
\hline ب & $\mathbf{b}$ & $\varepsilon$ & $\varepsilon$ \\
\hline ج & 3 & ف & $\mathbf{f}$ \\
\hline$د$ & d & ص & $\mathbf{S}$ \\
\hline 0 & $\mathbf{h}$ & ق & $\mathbf{Q}$ \\
\hline 9 & $\mathbf{w}$ & J & $\mathbf{R}$ \\
\hline j & $\mathbf{z}$ & ش ش & $\int$ \\
\hline$\tau$ & $\mathbf{h}$ & $ت$ & $T$ \\
\hline$b$ & $t$ & $\dot{H}$ & $\boldsymbol{\theta}$ \\
\hline ي & $\mathbf{j}$ & $\dot{\tau}$ & $\mathbf{X}$ \\
\hline ك & $\mathbf{k}$ & ض & d \\
\hline J & 1 & $\dot{\varepsilon}$ & б \\
\hline 5 & $\mathbf{m}$ & $\dot{ن}$ & $\mathbf{N}$ \\
\hline او & $\mathbf{u}$ & ق & $\mathbf{G}$ \\
\hline
\end{tabular}

\section{Autres procédés de transcription :}

1- La transcription phonétique entre [ ] uniquement pour l'arabe.

2- Les pauses sont indiquées par : // // (selon la longueur de la pose)

3- Les allongements vocaliques par : :: :: ::

4- Les intonations :

-Intonation ascendante :

-Intonation descendante :

5- Les sons para-verbaux sont indiqués entre parenthèses; exemple: (cris).

6- Voix off 1, 2,3,...: pour indiquer le commentaire dans chaque publicité Voix off (M) ou (F) : M pour masculin.

F pour féminin.

E pour enfant

7- Trad. : traduction du passage.

8- Le signe [...] présente des paroles coupées.

9- Les accolades \{\} pour les expressions en langues étrangères : anglais, tamazight, ...

10- Les guillemets pour tous les autres sons : animaux, balbutiements, propos incompréhensibles, ...

Pub1 (contexte algérien): Tramway Alger, durée : 43s Téléchargée le 29 novembre 2012 
Musique populaire en arrière plan tout au long du spot



Personnage 1 (H): [khlasrakum wasidn nwasalku:m ? ]

Personnage 2 (E) : ah papa [duka xlas]

Voix off $(\mathbf{H})$ : [talqa nasha kbar u søar taklin çla roћom ...]

Personnage 1 (H): [sbaћ xik nwaslak ? ] $\uparrow$

Personnage 3 (H) : [dorka khlas ja wlidi jactik asaha]

Voix off (H) : [ Jubanha naftin u mesawlin]

Personnage 4 (H) : [matabęatlif ] chauffeur [nji wahdi // dorka xlas]

Voix off $(\mathbf{H})$ : [cla raћma u lamћaba mrabjin...]

Personnage $5(\mathbf{H})$ : [nçawnak xuja ? ] $\uparrow$

Personnage $6(\mathrm{H})$ : [jactik asaha dorka xlas]

Voix off (H2) : tramway [alzazapir fi xidmatikum ibtidasan min tmanja maj]

Personnage 7 (voix off $\mathbf{H}$ ) : [ u ljum fi] tramway[alzazapir rana maztamein]

Pub2 : (contexte français) Réseau ferré de France, durée : 45s, téléchargée le

12 novembre 2012

Musique (paroles en anglais) tout au long du spot publicitaire

Voix off $\mathbf{H}$ : réseau ferré de France // construit le réseau de demain tout en faisant fonctionner celui d'aujourd'hui (musique du spot en arrière plan) //I/ (Slogan) : réseau ferré de France /// nous avons de grands projets pour vous

Pub3 : (contexte algérien) NEDJMA Sponsor officiel de l'équipe nationale de football (coupe du monde 2014), Durée : 53s, enregistrée la 10 juin 2012.

Chanson de Abdelhakim Baaziz

Paroles : [ki lbarah ki lyum // bladi candak riza :1 // jkun wa yku ::n waqfin dima mazal // waqfin dima mazal // // kamal mॄ̧ak dima naman bik // mॄ̧ak ja lxadra lqudam // NEDJMA fi sma balxir dawi clik // bin jadik amana laclam ///// meak ja lxadra // meak ja dzajar // meak ja lxadra// diri hala]

Personnages (joueurs de football de l'ancienne et la nouvelle équipe) : [mazal waqfin]

Pub4: (contexte français) EDF France sponsor des athlètes aux jeux olympiques de Londres 2012 , durée : 45s, Téléchargée le 17 décembre 2012.

Personnage $1(\mathbf{H}):$ ok :: on fait un test ?

Personnage 2 (H) : c'est parti

Musique sans paroles tout au long de la publicité

Personnage 1 (H) : salut :: // // / / // / / // // hé :: t'as vu

Personnage $2(\mathbf{H})$ : vas-y

Personnage 3(H) : alors ? ::

Personnage $2(\mathrm{H})$ : tout fonctionne

Personnage 3(H) : deuxième test?

Voix off $\mathbf{F}$ : les athlètes sont la lumière des jeux // nous sommes fiers de les éclairer. 
Pub5: (contexte français) Sécurité routière de France (le Gouvernement), durée: 58s, téléchargée le 23 mai 2013

Musique classique sans paroles comme arrière plan tout au long du spot publicitaire

Voix off $\mathbf{H}$ : Tant qu'il y aura trop d'alcool dans le sang d'un conducteur// il y aura du sang sur les routes // // / tant qu'un conducteur ratera un virage parce qu'il n'a pas voulu rater un appel // on appellera une ambulance // // tant qu'un véhicule de trois tonnes ignorera un scooter de cent kilos// il y aura des tonnes de dégâts // // // tant qu'on tournera sans faire attention à l'angle mort // il y aura des morts dans l'angle mort// // tant que la vitesse inscrite sur les compteur dépassera celle inscrite sur les panneaux // les accidents ne ralentiront pas // // tant qu'on continuera à penser que ce sont les autres qui conduisent mal //sans penser que les autres c'est nous tous // // tant qu'on comprendra pas que le code n'est pas là pour nous faire perdre des points de permis mais pour nous faire gagner des années de vie// // tant qu'il y aura des morts// il nous faudra agir pour une route plus sûre // // // // Tous responsables de la sécurité routière .

Pub6 : (contexte algérien) ALLO OTA (l'affaire du RAMADHAN), durée : 30 s, téléchargée le 07 février 2013.

Voix off 1(F) : [mą̧a] allo OTA[istafidu min xamsin bil mişa rasid iḍafi min shor laftor ]

Personnage 1 (l'apprenti): [aw! ya] inspecteur (haha) [ waqila olabha ramdan hadi lmaxluqa dork neajtalhom ]

Personnage 2 (l'inspecteur Tahar) : [aw ag̨aat ag̨̧at aw tkul u madiskitif bazaf yarhamaldik ]

Personnage 1 (l'apprenti): inspecteur [ya] inspecteur $\uparrow$ [asanani ya ] inspecteur

Personnage 2 (l'inspecteur Tahar) : [aw riti riti fi blastak yarhamaldik aw dana lgitha qablak ]

Voix off 1(F) : [likul tą̧ipa ibtidasan min mitin dinar] Allo OTA [tuhdikom

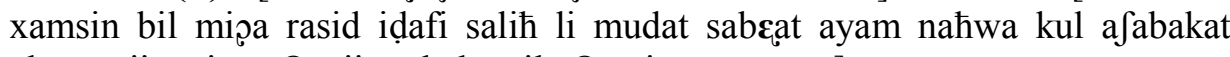
alwatanija mina aӨanija sabahan ila Đamina masapan]

Slogan (Voix off 1 F) : Allo OTA [sahl wa dakij]

Pub7 : (contexte français) la Box de SFR (internet), durée : $30 \mathrm{~s}$, téléchargée le

Chanson en anglais* $(0 \mathrm{~s} \rightarrow 20 \mathrm{~s})$

Voix off $\mathbf{F}+$ la chanson* (en double) : 
Avec SFR contrôlez et profitez de votre box à distance // // la box de SFR // la box qui n'a pas oublié d'être mobile // appelez le dix quatre vingt dix neuf

Pub8 : (contexte français) FREE Mobile Durée : 30 s, téléchargée le 18 mai 2013, diffusée sur W9 le 22 janvier 2012

Musique douce

Voix offF : forfait mobile [fri] // à deux euros ////// incroyable mais \{fri\}

Pub9 : (contexte algérien) Vénus (shampoing), durée : 34s, Téléchargée le 9 décembre 2012 Diffusée en décembre 2005

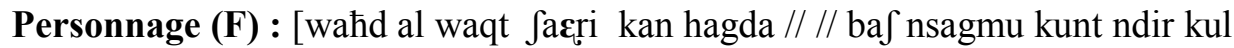
had $\int i \mathrm{u}$ nhar li staçmalt] shampoing Vénus deux en un [mawala ع̧andi hata mofkal // // bifadl tarkibtu li tradi u tlataf Ją̧ri //u tsahali lamfit tą̧u // // ana xajart ] shampoing Vénus deux en un [ u ntuma ?]

Slogan : Vénus [al 3ałnal al jawmi]

Pub10 : (contexte français) ELSEVE de L'OREAL PARIS (shampoing), durée : 30s, Téléchargée le 9 décembre 2012

Voix off $\mathbf{F}$ : (musique techno en arrière plan)

Vous aimez vos cheveux? les laissez pas tomber

Voix off $\mathbf{H}$ : révolution L'Oréal // ELSEVE arginine resist // découverte majeure l'arginine protéine indispensable à la croissance du cheveu pour une triple action renforçatrice // un :: nutrition du bulbe // deux :: renfort dès la racine// trois :: restructuration de la fibre// // les cheveux poussent plus résistants // / nouvel ELSEVE arginine \{résist \} de l'oréal Paris

Voix off F : Quatre vingt quinze pour cent des femmes ont constaté son efficacité.

Pub11 : (contexte français) Téléviseur SUMSUNG LED, durée : 30s Téléchargée le 17 décembre 2012

Voix off H : Qu'attendez-vous de la nouvelle génération? // Qu'elle se montre sous son meilleur jour // // // // / qu'elle respecte davantage ce qui l'entoure // // qu'elle ne cesse de vous séduire// // // // ou simplement qu'elle réunisse toutes ses qualités //

Slogan : LED tv la génération qui va tout changer.

Pub12 : (contexte français) Renault Mégane OVALIE dci 110, durée : 47, téléchargée le 17 mai 2012

(Drible de ballon)

Personnage $1 \mathbf{H}$ : bonjour :: vous n'auriez pas vu notre balon 
Personnage $2 \mathbf{H}$ : Quel ballon ::?

(musique et cris)

Personnage $2 \mathbf{H}$ : (sifflements [pssst])

Voix off $\mathbf{H}$ : en septembre tout Renault joue le jeu

Personnage $3 \mathbf{H}$ : bonjour

Voix off $\mathbf{H}$ : MEGANE OVALIE suréquipée est à quinze mille neuf cent quatre vingt dix euros.

Pub13 : (contexte français) la MAAF, durée : $35 \mathrm{~s}$, enregistrée le 25 novembre 2012

Personnage $1(\mathbf{H})$ : appelez-moi le directeur

Personnage 2 (H) : monsieur

Personnage $1(\mathbf{H}):$ ah $\uparrow$

Personnage $2(\mathbf{H})$ : ça ne va pas :: la MAAF na pas répondu à vos attentes ?

Personnage $1(\mathbf{H})$ : mais c'est-à-dire si :: $\uparrow$

Personnage 2 (H) : ben dans le pack de MAAF c'est écrit // vous pouvez nous évaluer :: content pas content ::c'est vous qui leldites

Personnage $3(\mathbf{F})$ : avec notre baromètre satisfaction :: notre remise en question est permanente $/ /$ mieux vous écouter ...

Personnage $2(\mathbf{H})$ : pour mieux nous améliorer

Personnage $1(\mathbf{H})$ : un assureur qui se fait noter par ses assurés?

Personnage $2(\mathbf{H})$ : oui c'est dans le pack de MAAF

Personnage 1 (H) : le « faktə paf ::knaf :: paf :: taf»

Personnage 2 (H) : éh stop euh :: autre chose?

Personnage 1 (H) : non

Personnage $2(\mathbf{H})$ : parc'que ::::

Personnage 1 (H): oui /// je l'aurais un jour :: je l'aurais // attends :: non ah non

Voix off (groupe) + musique : c'est laMAAF

Pub14 : [takdar tarbaђ] Trad. «Tu peux gagner» offert par SUMSUNG \& SAMHA

durée : 40s, enregistrée le 18 décembre 2012

Voix off (H) : [fi hisat takdar tarbah tą, lyum takadru tarbћu had al ] pack électroménager [li muhdat min taraf SAMHA wa SUMSUNG // // hada matkawan man] LED trente deux pouces marque 3D [nąam // furn kahrubapi ] encastrable [zapid had ] la table [atawila li tabx bil ]gaz [zapid had] la haute // // [asupal jakul // man huwa muwriz film arisala ? Lakhdar HAMINA // ibçatu raqm wahid // Mustafa ALAKAD ibcatu raqm tnin çan tariq al ] SMS [ bitabęa 






Pub15 : (contexte français) Question pour un champion (France 3), durée : 31s, enregistrée le 12 décembre 2012

Animateur : vous à la maison // là en ce moment où vous nous suivez :: quel est ce petit animal de compagnie qui stocke des aliments dans ses abajoues $\$ / /$ // c'est le chat :: réponse un :: // c'est le hamster :: réponse deux :: // donc vos réponses sur le trente deux quarante cinq maintenant // ya pas d'autres numéros c'est pas la peine de chercher //c'est le trente deux quarante cinq // et ça passe par ce seul et unique numéro // trente deux quarante cinq :: trois mille euros en jeux // tirage au sort demain // parmi les bonnes réponses bien sur // // donc la réponse un ou deux // trente deux quarante cinq toute de suite $[. .$.

Pub16 : (contexte français) ORANGE (Open : Internet-tv-téléphone +mobile), durée : 56s, téléchargée le 07 février 2013

\section{Voix offH (doublage du père noël en bougie) :}

Bon :: ben j'étais au pied du sapin :: [bb]pas trop près pour éviter les dégâts // je m'étais mis sur mon trente et un // enfin je veux dire sur mon vingt cinq (rire ) nfon vingt cinq // oui bon et et :: là ils ouvrent leurs cadeaux et hop un téléphone et hop encore un téléphone et encpre un autre // ça n'arrêtais plus :: alors sur le coup j'ai cru que c'étais un coup de show :: que j'avais division mais en fait pas du tout :: c'était une sorte de super cadeau commun // [oh] à les voir tous réunis // ça m'a fait fondre [pask] moi les copains je les vois qu'une fois par an et et encore pas longtemps // avec un truc pareil on pourrait au mois rester en contact :: pas vrai les gars? Les gars ${ }^{\Uparrow}$ (Sons d'tne chouette) (Musique courte)

Pub17 : (contexte algérien) Café Many, durée : 31 s, téléchargée le 25 septembre 2012.

Musique calme et des renflements

Paroles de la chanson : [mani mani nfani mani mini ma :::: ni mani :: // qahwat mani duqha ją̧зabnisirhijali tafhamni //// męa hbabi u sirani sirhija li tafhamni ]

Pub18 : (contexte algérien) ACTIVIA de DANONE, Durée : 25 s,téléchargée le 16 mai 2012

Musique algérienne populaire en arrière plan

Personnage 1 (F) : [ana fi ramdan dari tahja ki jkunu wladi dajrin bija //

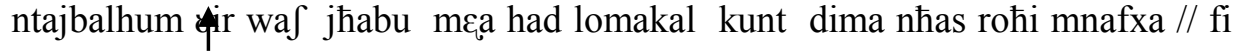




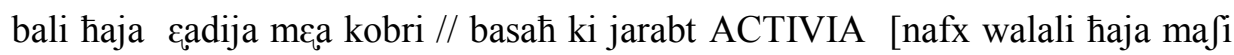
દadiya] (mmm)

Voix off (F) : [ bifadl el] bifidus ACTI régularise ACTIVIA [jusą̧id çala muharabat anafx]

Personnage 1 (F) : ACTIVIA [rajaћni u xalani natmata

(Slogan) Voix off $(\mathbf{H})$ : ACTIVIA [afdal halif ḍida anafx]

Pub19 : (contexte français) ACTIVIA de DANONE, durée : 30s, enregistrée le 10 octobre 2012

Voix off $\mathbf{F}$ : pourquoi commencer l'année avec le programme Activia?

Personnage $1(\mathbf{H})$ : pour nous (personnage $2 \mathbf{F}$ : ah chéri) les fêtes ça commence un mois avant $::$ y a le repas de noël de bureau (personnage $2 \mathbf{F}$ : $\mathrm{mm}$ ) des beaux parents (personnage $\mathbf{2 F}$ : et les tiens tu n'oublies pas :: $\}$ oui et les miens aussi demain // on n'en sort pas de table près d'un mois (personnage $2 \mathbf{F}$ : du coup après on se sent un peu ballonné// alors on a essayé le programme Activia un peu de sport tous les jours\} wé !! (Personnage 2 F : si // si si // des repas équilibrés et Activia tous les jours) Activia ça ! Tous les jours \{personnage $2 \mathbf{F}$ : en quinze jours ça $\mathrm{m}$ 'a aidé à mettre de l'ordre à l'intérieur // $\mathcal{E}^{\prime}$ ) ah franchement je confirme // celui aux manguess là :: j'aime beaucoup (personnage $\mathbf{2} \mathbf{F}$ : ah oui enfin :: beaucoup beaucoup quoi )

Voix off $\mathbf{F}$ : Essayez Activia ::en ce moment c'est convaincu ou remboursé.

Pub20 : (contexte algérien), Réfrigérateur ENIEM, durée : 31s,téléchargée le 12 novembre 2012

Personnage 1 (F) : [min raki jibi had lma lbarad u] les glaçons [kamal ? // qulili wa h hwwa sir djalak? ]

Personnage 2 (F) : [sir djali ? Galazat] \{side by side\} ENIEM [bzihtin // fiha

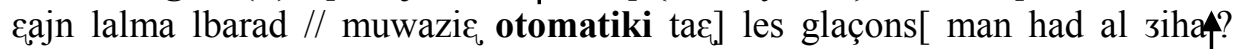

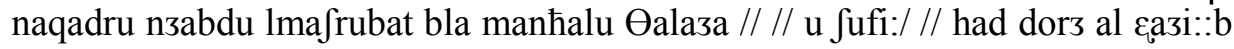
naqdar nathakam fih kima nhab fi daraza tą̧u// u lhaza li thotiha fih tabqa 3dida]

Personnage 1 (F) : [lazamli ana wahda kima hadi fi dari]

(Slogan) Voix off (F): [mॄa] ENIEM [tahla al ajam]

Pub21: (contexte algérien), DJAWEB d'Algérie télécom (modem de connexion), durée : $37 \mathrm{~s}$ ), téléchargée le 28 décembre 2012

Musique sans paroles tout au long de la pub

Voix off (H) : [athana man xjot al ] internet[ fi darak :: al jawm megalçard al

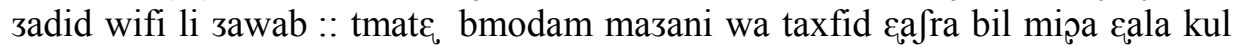
iftirak xamsamja u ṭa u wahad] méga :: ::::: [ mąa al wifi ta tmata , bi huriat alwab ]

Slogan: [itisalat al zazajar :: al ixtijar al amӨal] 
Pub22: (contexte algérien), BlackBerry de Mobilis (offre de téléphonie mobile), durée : $41 \mathrm{~s}$, Téléchargée le 12 novembre 2012

Musique sans paroles tout au long de la publicité

31 s $\rightarrow$ Voix off $(\mathbf{H}):$ [muћtarifun aw xawas :: iktafifu] BlackBerry [ li mobilis waxtarou ahad alçardajn almutamajizajn]

Slogan : [mobilis wal kulu jatakalam]

Pub23 : (contexte algérien) OMO d'UNILEV, durée : 30s, enregistrée le 29 novembre 2012

Musique sans paroles

Voix off $(\mathrm{H})$ : [fi bacd al halat asąba hata lqamis lomosax jaqdar ykun adqa 1hulul // // // u latafat asąba makalah tastąmli Jita wala sabun hajra // OMO

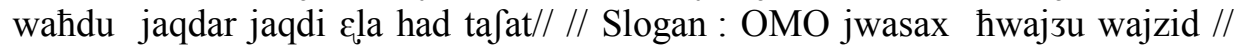
jatçalam huwa al mufid] 